\title{
A NEW MODEL FOR INTEGRATING SERVICES, INFORMATION AND PEOPLE: U-GOV INTEGRATION, DISSEMINATION \& COLLABORATION
}

Antonio Storino, Federico Gallerani

Keywords

Soa, integration, cloud services, governance, social network, esb, service registry, business process , anagement, erp, data integration, identity management, authentication and authorization , anagement, lifecycle management, unified search, elastic architecture, open standards, master data management.

\section{ABSTRACT}

U-GOV Integration, Dissemination \& Collaboration is a solution to connect people to data and services.

It brings into the higher education institutions an innovative model of integrated work and a generation of completely new services that help making interactions more efficient and flexible.

It has three main aspects: Identity and Service Management, Business Process Management and Integrated \& Collaborative Services.

\section{Identity and service management}

The Identity and Service Management is focused not only on the concept of "user" but on that of "person": a person is unique but can act according to multiple profiles, in different contexts and at different times. Our solution can offer such an opportunity thanks to the ease of management and a very low number of manual operations.

It manages both the "person governance" and the "services governance". This functionality allows our solution to centralize a management process which is usually fragmented (user profiles replicated for each single service, lack of a service registry within the institution, manual operations, etc.).

It is a completely new way to manage identities and services' authorizations, with a more structured and integrated approach: the person is connected to the institution's services on the basis of its general policies and authorization rules for people. It is easier to modify and adapt the levels of authorization (i.e. the student administration's services, project developments, research outputs, wi-fi access, print services and others) depending on the people needs and their specific roles, which can change over time within the institution.

The Identity and Service Management includes Governance tools to gather and analyze data about the quality of services offered, by monitoring and auditing the system's and service's performance and the user's perception and satisfaction.

\section{Business Process Management}

The Business Process Management platform included in the U-GOV solution provides an institution with the right tools to set cross processes and communicate with internal and external systems and connect data or applications which can be internal and in the external cloud.

The solution simplifies the human tasks and reduces human mistakes during the implementation of automatic processes and dynamic User Interfaces depending on the processes' layer. 
Thanks to it, an institution is able to easily manage not only the processes operating within the same institution, but also those involving communications between different institutions. To change the logic behind a process there is no more need of a developer, because it can be easily adjusted by the analyst in few "mouse clicks" without changing the source code.

\section{Integrated \& Collaborative Services}

The U-GOV solution provides appropriate technologies to manage communication among persons and groups, research and learning activities, deadlines within complex organizations and provides social networking tools. Such services manage the informal communication within the institutions and are complementary to the formal communication processes.

One of the main features is the management of "work-groups". Within a work-group, people can communicate easily, share a pool of centralized information, and cooperate using shared and standard tools, or using external tools as well.

Such approach makes the solution able to achieve an important goal: to connect people and to connect them to processes and information, working together and beside the management platforms.

\section{Service Integration and Delivery Platform}

The solution is based on a SOA (Service Oriented Architecture) architecture.

Applications and services lie on an integrated layer formed by an enterprise service bus and several connectors toward data and service providers. This specific architecture with different components helps to decouple services and makes it easy to re-use them. As a consequence, services and applications follow a plug-in model and they do not depend on the technological platform. This allows the single institution to customize and redistribute them as they wish.

The solution is based on a mix of open source platforms, Google Search Appliance and Groovy on Rails open-source frameworks. In 2013-2014 it will be moving from an Oracle BEA platform to a full open source one. One of the pillars of this solution is the use of standard technologies and protocols.

It is offered as a multi-tenant service that can be used on a SaaS paradigm. Starting from 2013 it will be available as PaaS too, to develop new services on top using API, web services, Registry/Repository, standards technologies/protocols and other components of the SOA platform and our built-on-top layers.

It is easy to customize for specific needs and can be configured to run as multi-company (i.e. universities, research centers) and can be federated by design.

Other than that, it is natively developed to cover a national academic and private research ecosystem.

\section{Technological Overview}

Supported standards include: XML, OASIS BPEL, OASIS WS-Security, JSR-168 and JSR-286, WSRP, SCA, SOAP, JCA, JMS, JPA, XSLT/XQuery, WS-Policy, SAML, WSDL/WSIF, X509, JKS, XACML, WSDL, UDDI, EJB, HTTP, JMX, XA.

Authentication management: it is possible to synchronize it with a central repository, like an LDAP and/or Active Directory, or with separate sources. In the latter case the separate repositories are exposed as a single virtual repository.

Authorization management: it is possible to manage roles and user profiles to control the access to applications and services during the whole user life cycle.

Application interfaces (Web Services, for example): WS-Security and others security protocols and models are available by ESB mediation as well and STS for authentication and endpoint single signon.

There are several SSO authentications modes available: SAML (Shibboleth used protocol), CAS, OpenID, Kerberos, OAuth and other platform-specific modules. Format of editions and data exports can change depending on different requirements without any impact on complexity: data 
transformation and formatting depend on specific ESB capabilities. Data can be stored in databases, file systems, document management systems through JSR-170, WebDAV and other protocols. Different information systems can be connected to the application in different modes: multiprotocol, direct, batch or transactional. CERIF is currently in evaluation.

\section{AUTHORS' BIOGRAPHIES}

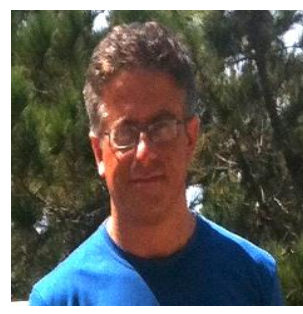

\section{Antonio Storino}

As Integration Division Head for CINECA Storino is responsible for integration projects for Universities as well as leading the division product development and technology strategy for next-generation services.

Before dealing with integration, he introduced the enterprise portal in CINECA and changed the pattern of delivering services in some of the largest universities in Italy.

Prior to joining CINECA, Antonio was Chief of Innovation Officer for a company of an important Italian industrial group and has designed new generation services for teaching. Before that he created services for Italian banks and for the UK health system.

Antonio studied computer science, Pisa and general management at Alma Graduate School, Bologna.

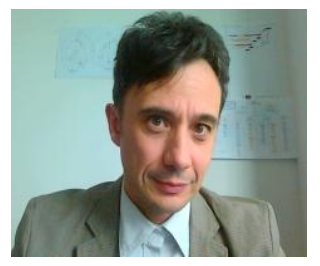

\section{Federico Gallerani}

Gallerani, as CINECA's technologist, is responsible for the U-GOV Solution (Integrated System for University GOVernance) and coordinates the Product Manager team.

He has been working in the field of Information Systems for Higher Education and interoperability. since 2003, he has participated in several projects regarding system integration 